\title{
Correction: Towards a deeper understanding of parenting on farms: A qualitative study
}

The PLOS ONE Staff

\section{Notice of republication}

An incomplete, earlier version of this manuscript was published in error. The publisher apologizes for the error. This article was republished on August 22, 2018 to correct for this error. Please download the article again to view the correct version. The originally published, uncorrected article and the republished, corrected article are provided here for reference.

\section{Supporting information}

S1 File. Originally published, uncorrected article.

(PDF)

S2 File. Republished, corrected article.

\section{Reference}

1. Elliot V, Cammer A, Pickett W, Marlenga B, Lawson J, Dosman J, et al. (2018) Towards a deeper understanding of parenting on farms: A qualitative study. PLoS ONE 13(6): e0198796. https://doi.org/10. 1371/journal.pone.0198796 PMID: 29897960

\section{G OPEnACCESS}

Citation: The PLOS ONEStaff (2018) Correction: Towards a deeper understanding of parenting on farms: A qualitative study. PLOS ONE 13(9): e0203842. https://doi.org/10.1371/journal. pone.0203842

Published: September 6, 2018

Copyright: @ 2018 The PLOS ONE Staff. This is an open access article distributed under the terms of the Creative Commons Attribution License, which permits unrestricted use, distribution, and reproduction in any medium, provided the original author and source are credited. 